\title{
Riscos psicossociais relacionados ao trabalho do enfermeiro da Saúde da Família e estratégias de gerenciamento
}

\author{
Work-related psychosocial risks of the Family Health nurse and management strategies \\ Riesgos psicosociales relacionados con el trabajo de los enfermeros \\ de la Salud de la Familia y las estrategias de gestión
}

Como citar este artigo:

Celestino LC, Leal LA, Lopes OCA, Henriques SH. Work-related psychosocial risks of the Family Health nurse and management strategies. Rev Esc Enferm USP. 2020;54:e03602. doi: https://doi.org/10.1590/S1980-220X2018055603602

\section{Lázaro Clarindo Celestino ${ }^{1}$ \\ Laura Andrian Leal ${ }^{2}$ \\ Olivia Cristina Alves Lopes ${ }^{1}$ \\ Silvia Helena Henriques ${ }^{3}$ \\ ${ }^{1}$ Universidade de São Paulo, Escola de Enfermagem de Ribeirão Preto, \\ Programa de Pós-Graduação Mestrado Profissional em Tecnologia e Inovação em Enfermagem, Ribeirão Preto, SP, Brasil. \\ ${ }^{2}$ Universidade de São Paulo, Escola de Enfermagem de Ribeirão Preto, Programa Interunidades de Doutoramento em Enfermagem, Ribeirão Preto, SP, Brasil. \\ ${ }^{3}$ Universidade de São Paulo, Escola de Enfermagem de Ribeirão Preto, \\ Departamento de Enfermagem Geral e Especializada, Ribeirão Preto, SP, Brasil.}

\section{Autor correspondente:}

Laura Andrian Leal

Escola de Enfermagem de Ribeirão Preto, Universidade de São Paulo

Av. dos Bandeirantes, 3900, Campus Universitário, Bairro Monte Alegre CEP 14040-902 - Ribeirão Preto, SP, Brasil laura.andrian.leal@usp.br

\begin{abstract}
Objective: To analyze the work-related psychosocial risks of Family Health nurses and the management strategies to minimize them. Method: Exploratory, qualitative study with nurses from teams of the Family Health Strategy (FHS). Data collection took place in July 2018 through semi-structured interviews and subsequently, an inductive thematic analysis was carried out. Results: Participation of 18 nurses. Psychosocial risks related to the work context were identified, namely: insufficient professional training, compromised interpersonal relationships, work-family interface and psychological violence; as well as those related to work content: insufficient work equipment, lack of human resources and heavy workload. In addition, strategies for managing these risks were identified, such as using the family, spirituality, music and reading. Conclusion: The study should provoke the reflection of nurses about the eventual presence of risks in their work and contribute to fill knowledge production gaps in the area. It also provides subsidies for health managers' knowledge of the psychosocial risks to which these professionals are exposed, with a view to adopting measures.
\end{abstract}

\section{DESCRIPTORS}

Family Nursing; Family Health Strategy; Occupational Risks; Primary Care Nursing; Occupational Health. 


\section{INTRODUÇÃO}

O processo de reestruturação da atenção básica no país, por meio da Estratégia de Saúde da Família (ESF), repercutiu significativamente na atenção à saúde da população através da adesão a estratégias para melhorar o atendimento básico, aperfeiçoar o fluxo de atendimento e proporcionar tratamentos efetivos com maior eficiência, gerando satisfação dos usuários e aumentando a resolubilidade deste serviço ${ }^{(1)}$.

As equipes da ESF são compostas por trabalhadores de distintas categorias, dentre as quais o profissional enfermeiro se destaca como protagonista e responsável pelas atividades desenvolvidas pelos demais membros da equipe. A ele são atribuídas diversas funções, tais como: planejar e executar ações no âmbito da saúde coletiva; supervisionar a assistência direta a população; gerenciar os serviços de saúde; gerenciar e coordenar as atividades desenvolvidas pelos Agentes Comunitários de Saúde (ACS); coordenar e realizar atividades de educação permanente; participar ativamente do gerenciamento dos insumos necessários para o bom funcionamento da ESF, dentre outras ${ }^{(2)}$. A complexidade das atividades executadas pelo enfermeiro, somadas às condições de trabalho inadequadas, podem expor este profissional a riscos ocupacionais, dentre eles, os psicossociais.

A título de exemplo, a jornada de trabalho pode ser citada como uma condição inadequada que afeta enfermeiros da ESF. Pesquisadores apontam que a jornada de trabalho excessiva eleva significativamente as cargas de trabalho destes profissionais em decorrência dos problemas relacionados às condições de trabalho, excesso de demanda, estrutura física inadequada e insatisfação com salários. As cargas de trabalho são elementos presentes no dia a dia do processo de trabalho dos enfermeiros, interagem entre si e com o corpo de quem executa o trabalho, e em grande parte das vezes, levam o indivíduo ao desgaste físico e emocional ${ }^{(3)}$.

Os riscos psicossociais são definidos como aqueles relacionados à concepção, organização e gestão do trabalho ${ }^{(4)}$. Assim, derivam da forma como o trabalho está organizado nas instituições e da interação de seu conteúdo, sendo que a exposição dos trabalhadores a estes riscos podem gerar prejuízo físico, mental ou social aos trabalhadores ${ }^{(4)}$ e representar um grande desafio para a segurança e saúde ocupacional ${ }^{(5)}$.

A literatura também demonstra que entre os profissionais de saúde, os enfermeiros da ESF são os mais afetados por problemas psicológicos pelo fato de suas atribuições serem em grande parte, determinadas por metas de programas do Ministério da Saúde. Isso limita o profissional frente aos problemas da população como um todo e interfere na agenda de demanda espontânea, deixando-o inseguro, o que gera sentimento de impotência, desgaste físico e emocional, caracterizando exposição a riscos psicossociais ${ }^{(6-7)}$.

Riscos psicossociais relacionados ao trabalho de profissionais da ESF já foram identificados em outros estudos, como a sobrecarga de atividades, longas jornadas de trabalho, falta de preparo e capacitação, sobrecarga de papéis, longas horas de trabalho e recursos materiais insuficientes ${ }^{(8-9)}$. Em breve pesquisa na literatura científica dos últimos cinco anos, nas bases de dados LILACS (Literatura Latino-Americana e do Caribe em Ciências da Saúde) e CINAHL (Cumulative Index to Nursing and Allied Health Literature), utilizando descritores como "enfermeiros", "saúde da família" e "riscos ocupacionais", e a palavra chave "riscos psicossociais", não foi identificado estudo cujo objeto de pesquisa fosse somente o profissional enfermeiro da Saúde da família.

A exposição aos riscos psicossociais pode acarretar consequências negativas a nível fisiológico e psicológico para a sociedade, instituição e para a saúde dos trabalhadores. Contudo, os efeitos cruciais estão relacionados às consequências organizacionais e individuais ${ }^{(10)}$.

Considerando o processo de trabalho do enfermeiro nas diferentes áreas de atuação, em especial na ESF, é relevante olhar para aspectos que interferem na sua saúde, identificar suas fragilidades, potencialidades e possibilidades de melhora. O conhecimento teórico e empírico da realidade da ESF tem revelado condições de trabalho diversas e, muitas vezes, precárias para o enfermeiro. Tais condições, somadas às exigências de suas atividades, a complexidade da realidade sanitária, e a forma como seu processo de trabalho está organizado, podem expor este profissional a riscos psicossociais. Assim, esse estudo apresenta as seguintes questões norteadoras: Quais são os riscos psicossociais a que os enfermeiros da ESF estão expostos? Quais estratégias podem ser utilizadas para gerenciar os riscos presentes?

Este estudo teve o objetivo de analisar os riscos psicossociais relacionados ao trabalho do enfermeiro da ESF e as estratégias de gerenciamento para minimizá-los.

\section{MÉTODO}

\section{TIPO DE ESTUDO}

Trata-se de um estudo exploratório de abordagem qualitativa dos dados.

\section{Cenário}

O cenário do estudo foi composto por 18 unidades da ESF de uma cidade do interior de Minas Gerais. As 18 Unidades da ESF estão distribuídas em pontos estratégicos da cidade e são responsáveis por território sanitário definido, com aproximadamente 3.000 pessoas para cada equipe. A época da coleta de dados, o município possuía 18 equipes de ESFs e todas aceitaram participar da pesquisa.

\section{CritérIos de SELEÇ̃̃o}

Enfermeiros do referido município, exercendo suas atividades há mais de três meses do início da coleta de dados, uma vez que esse tempo de trabalho deve possibilitar a este profissional experiências para identificar os riscos psicossociais presentes no trabalho.

\section{Colleta De Dados}

Para a coleta de dados, foram realizadas entrevistas semiestruturadas gravadas em áudio e posteriormente transcritas, além de roteiros validados com enfermeiros especialistas da área. A amostra foi definida por saturação dos dados, ou seja, o momento em que o acréscimo de dados e 
informações em uma pesquisa não altera a compreensão do fenômeno estudado, permitindo estabelecer a validade de um conjunto de observações ${ }^{(11)}$. O roteiro continha duas partes: a primeira com questões referentes a dados de caracterização dos participantes, tais como idade, sexo, ano de conclusão da graduação e tempo de atuação na instituição. $\mathrm{Na}$ segunda parte, havia questões abertas relacionadas ao objeto deste estudo, tais como: No desempenho de suas atividades, que condições são oferecidas pelo serviço? Existe alguma dificuldade? Como você se sente após uma jornada de trabalho? $\mathrm{O}$ que você considera desgastante e prejudicial para sua saúde? Como é para você conciliar as atividades e o tempo de dedicação ao trabalho e à sua família? Você se utiliza de algum recurso pessoal ou institucional para aliviar os efeitos de situações difíceis que enfrenta no dia a dia do seu trabalho?

$\mathrm{O}$ contato com os enfermeiros para agendamento das entrevistas foi feito por telefone (números repassados pela coordenação da Atenção Primária). As entrevistas foram realizadas na própria unidade de Saúde da ESF de cada enfermeiro, pelo pesquisador responsável com o intuito de evitar viés, em horários estabelecidos pelos profissionais enfermeiros, individualmente, sem nenhuma interrupção e duraram cerca de 20 minutos.

\section{ANÁLISE E TRATAMENTO DOS DADOS}

Os dados foram analisados segundo análise temática indutiva e organizados em categorias temáticas seguindo três etapas: pré-análise, exploração do material e tratamento dos resultados obtidos. Para a identificação das unidades temáticas relacionadas aos riscos psicossociais, foram levados em conta os discursos dos entrevistados à luz do referencial teórico utilizado ${ }^{(12)}$.

\section{Aspectos étIcos}

O estudo foi aprovado pelo Comitê de Ética em Pesquisa da Escola de Enfermagem de Ribeirão Preto da Universidade de São Paulo, sob o protocolo Ofício n ${ }^{\circ}$ 090/2018. Todos os participantes assinaram o Termo de Consentimento Livre e Esclarecido e foi assegurado o sigilo das respostas. Visando preservar o anonimato dos participantes desta pesquisa, optamos por utilizar a letra "E" de entrevistado, seguida do numeral, de acordo com a ordem cronológica crescente da realização das entrevistas, por exemplo, E1 (Entrevistado 1), e assim sucessivamente.

\section{RESULTADOS}

\section{CARACTERIZAÇÃO dos PARTICIPANTES}

Participaram do estudo 18 enfermeiros, com predomínio do gênero feminino 16 (89\%) e da faixa etária de 36 a 40 anos, o que correspondeu a 12 profissionais (67\%). O tempo de formação universitária predominante foi de 15 anos, bem como de atuação na ESF. Havia profissionais atuando na área de saúde da família desde o início de sua formação profissional.

Quanto à capacitação, 12 (67\%) profissionais possuíam nível de pós-graduação, dos quais, todos com título de especialização lato sensu e 2 (11\%) com título de mestrado (stricto sensu). Foram encontrados profissionais com até cinco títulos de especializações, o que reforça o perfil de trabalhador de enfermagem capaz de atender a diversidade de desafios presentes na ESF.

A carga horária de trabalho dos profissionais enfermeiros foi de 40 horas semanais e oito horas diárias. Ao analisar os dados das entrevistas, foram identificados diversos riscos psicossociais relacionados ao contexto do trabalho do enfermeiro da saúde da família, distribuídos em quatro categorias: capacitação profissional, relacionamento interpessoal, interface trabalho e família, violência psicológica. Houve também riscos psicossociais relacionados ao conteúdo do trabalho do enfermeiro da ESF, distribuídos em três categorias: equipamento de trabalho, recursos humanos e carga e esquema de trabalho. Além disso, foram identificadas estratégias para gerenciar tais riscos.

RISCOS PSICOSSOCIAIS RELACIONADOS AO CONTEXTO DO TRABALHO DO ENFERMEIRO DA SAÚDE DA FAMÍLIA

\section{Capacitação profissional insuficiente}

Os enfermeiros revelaram ausência de investimentos em capacitação profissional e o aprendizado acontecendo no cotidiano do trabalho da saúde da família, o que configura um risco psicossocial relacionado ao trabalho.

Eu não tive nenhum preparo. Foi tudo assim, aprendendo no dia a dia mesmo. Fui lendo, estudando, porque não teve nenhum treinamento, nenbum curso, nem nada (E18).

Relacionamento Interpessoal: dificuldade de relacionamento com colegas de trabalho e a gestão, conflito interpessoal no trabalho da equipe

A dificuldade de relacionamento entre os profissionais e com a população pode levar o profissional ao desgaste emocional e, assim, ser percebido como um risco psicossocial: $O$ relacionamento com a equipe e a população como um todo é muito desgastante. (...) E com a gestão também, ainda tem questões politicas que mexem negativamente, então isso é muito ruim. Fico muito chateada. Mas, com o passar do tempo aprendi a passar um filtro, senão fica insustentável (E3).

Tem a técnica de enfermagem que é muito difícil, ela não dá certo com ninguém da equipe. (...) O médico também é bem inflexivel, não cumpre o horário dele (E18).

Interface trabalho e família: dificuldade de conciliar as exigências do lar com as do trabalho, pouco tempo dispensado à família

As exigências do trabalho somadas às tarefas domésticas e o pouco tempo dispensado à família podem configurar um risco psicossocial, se não houver equilíbrio entre elas:

Não consigo me dedicar o suficiente à minha família. Passo mais tempo aqui na unidade do que com eles. Eu tento suprir esta falta no final de semana, sair com os filhos, marido, mesmo assim é difícil, porque eu acabo levando serviço para casa (E11).

(...) Não consigo conciliar. Eu tenho filho, marido. No horário de almoço, dou um pulinho lá pra almoçar com eles. (...) O tempo é muito curto (E5). 
Violência psicológica: exposição excessiva dos enfermeiros na mídia

Os enfermeiros percebem que dependendo de suas atividades ou reações diante do usuário, pode haver certo repúdio por parte da mídia, às vezes, fora de propósito, representando um ato de violência psicológica, o que é considerado um risco psicossocial importante para a saúde mental do trabalhador. Nesta cidade, a comunidade tem o costume de reclamar do enfermeiro na mídia, na rádio, mete broncas nas páginas do Facebook. Eles não procuram saber o que aconteceu de fato, vai logo metendo bronca no profissional e expondo na mídia, é um jogo muito sujo. (...) Eu me sinto violentada psicologicamente (E6).

\section{RISCOS PSICOSSOCIAIS RELACIONADOS AO CONTEÚDO DO TRABALHO DO ENFERMEIRO DA SAÚDE DA FAMÍLIA}

Equipamento de trabalho: recursos materiais insuficientes e falta de manutenção

Os discursos revelaram que o ambiente de trabalho apresenta grandes limitações e falta de manutenção, onde os trabalhadores precisam improvisar recursos materiais para realizar suas tarefas.

Nossas condições são de improviso. Falta material básico de uso, mobilia. Falta esfignomanômetro, estetoscópio, sonar, foco, computadores. Os materiais vão para a manutenção, porém demora muito, dai fica tudo desatualizado, principalmente quando os computadores estragam (E10).

(...) Falta muito material, inclusive o carro para deslocar até a casa do cliente para realizarmos a visita domiciliar. Às vezes é um local longe, mas, mesmo assim não tem carro e temos que usar o nosso pessoal. (...) Falta PVI tópico, medicamentos, até dipirona (E6).

Recursos humanos: déficit no quadro de pessoal e desvio de função

O número insuficiente de profissionais e o desvio de função dos trabalhadores que compõe a ESF foi revelado nos discursos dos enfermeiros como um risco psicossocial:

Minha equipe está totalmente incompleta. Sou eu [enfermeira], a médica e apenas duas agentes de saúde nesta unidade" (E17).

O 70 agente de saúde está com desvio de função, ele fica na recepção. E a nossa auxiliar de limpeza só vem duas vezes na semana. Eu acredito que uma unidade de saúde precisa de uma auxiliar de limpeza no minimo de 3 vezes por semana (E13).

Carga e esquema de trabalho: sobrecarga e longas horas

Os relatos mostraram que os enfermeiros executam grande número de atividades no seu cotidiano e ainda levam serviço para casa, além de ultrapassarem sua carga horária de trabalho habitual da ESF.

Geralmente, meu horário de sair daqui é 16:30 h, mas eu sempre vou embora às 17:00 h, 18:00 h pois tem muito serviço. (...) Eu me sinto super cansada, eu sempre levo serviço pra casa, principalmente na época de fechamento de produção (E14).

Estratégias de gerenciamento dos riscos psicossociais

A presença de riscos psicossociais revela a necessidade de elaborar estratégias para combatê-los. Apesar disso, os discursos dos enfermeiros mostram a ausência total de atividades ou ações oferecidas pela coordenação das equipes da ESF para eliminar ou minimizar os riscos analisados:

Por parte da instituição, não. Eles não oferecem nenbum recurso ou estratégia para gerenciar os riscos que estamos expostos (E3).

Não existe nenhum apoio ou ação da instituição neste sentindo, principalmente esta coordenação atual, eles não se preocupam, ainda mais com algo para nos ajudar (E14).

Entretanto, estratégias individuais foram evidenciadas pelos profissionais para amenizar situações de risco, tais como prática de atividade física, ajuda espiritual, terapia com psicólogo e meditação:

Eu faço terapia com o psicólogo e meditação, pois me ajuda muito(E1).

Eu procuro fazer atividade física, no minimo quatro vezes na semana, pois é o que me ajuda a aliviar um pouco o estresse. Eu não abro mão desta estratégia, senão eu surto (E15).

Eu utilizo como estratégia a espiritualidade. Eu sempre busco ajuda neste sentido, sou cristã de muita fé. Isso me ajuda muito, melhora minha disposição para o trabalho, minha saúde, é um bem-estar, né(E7).

\section{DISCUSSÃO}

A implantação da ESF com o enfoque da promoção da saúde e prevenção de doenças e a proposta de reorganizar a atenção básica no Brasil, corroborou para um exército de trabalhadores em condições precárias de trabalho. Assim, os riscos ocupacionais ganharam grande proporção, em especial os psicossociais ${ }^{(8-9)}$.

Esta investigação revelou a falta de capacitação profissional como um risco psicossocial para os trabalhadores, corroborando com outro estudo em que este aspecto também foi considerado um risco para trabalhadores de enfermagem ${ }^{(9)}$ e demonstrando a relevância da formação profissional para o cuidado em saúde. Os dados mostraram evidências da ausência de investimentos das instituições para a formação e preparo dos enfermeiros para atuação na ESF. A realidade sanitária complexa deste cenário requer profissionais atualizados e preparados, com competências específicas para atender às demandas dos usuários dos serviços que se modificam constantemente.

Capacitar é preparar/habilitar o trabalhador para desempenhar determinada função. A possibilidade de capacitação deve ser real no âmbito dos serviços de saúde e acontecer logo após a contratação dos servidores. Além disso, é fundamental que as instituições de Saúde tenham seus objetivos bem definidos e trabalhadores seguros para executarem a função que lhes foi $\operatorname{confiada}^{(9)}$.

Nesse aspecto, é imprescindível que as instituições de saúde assumam sua responsabilidade e invistam em treinamentos e capacitação de seus profissionais. É relevante que este preparo seja oferecido antes da inserção do profissional no mercado de trabalho e não depois de estar atuando na função por anos. Os centros formadores em enfermagem têm o papel de desenvolver estratégias de ensino durante a formação acadêmica para auxiliar no desempenho dos futuros enfermeiros e esclarecer as reais condições de trabalho, de 
forma que os profissionais possam atuar com efetividade nos diversos serviços de saúde da complexa realidade sanitária.

O relacionamento interpessoal é um dos grandes problemas nas equipes da ESF e considerado um fator de risco psicossocial. Neste estudo, foram identificadas discussões calorosas no cotidiano do trabalho dos membros da equipe. Os enfermeiros relataram conflitos com os profissionais médicos e o principal motivo tinha relação com o não cumprimento da carga horária preconizada e atrasos constantes. Também há confronto entre a própria equipe de enfermagem, os agentes comunitários e a população como um todo.

Em consonância com esta pesquisa, autores afirmam que a saúde é um campo complexo e frágil, onde ocorrem diversos conflitos devido às transformações no perfil epidemiológico e demográfico, pelas relações de poder entre os membros da equipe multidisciplinar e outros determinantes sociais ${ }^{(13)}$. Estas transformações e fatores vislumbram a necessidade de um trabalho coletivo da ESF, o que na maioria das vezes é tarefa difícil pelos movimentos tensionados que norteiam as equipes de Saúde da Família. A harmonia e interação entre os profissionais da ESF é fundamental para sua atuação de forma integrada ${ }^{(14)}$.

O desequilíbrio no ambiente de trabalho, ou a falta de harmonia entre os próprios profissionais podem até mesmo gerar exposição desnecessária do trabalhador, causada por manobras e intrigas de uma minoria. Este fato é percebido como ato de violência psicológica no trabalho e assim, uma condição de risco psicossocial. Os discursos dos enfermeiros revelaram situações de violência vivenciadas por eles no cotidiano laboral e destacaram como agravante, a exposição do enfermeiro em mídias como rádio e redes sociais. Além disso, revelaram ainda a falta de interesse e apoio da gestão na proposição de soluções para diminuir a exposição dos profissionais nas redes sociais.

Em consonância com o apresentado, estudo recente identificou a violência psicológica no trabalho do enfermeiro como risco psicossocial e destacou como formas dessa violência a agressão verbal, assédio moral e sexual. As mulheres e os profissionais mais jovens foram os mais atingidos pela violência; e os pacientes e familiares foram os maiores agressores destes profissionais. Além disso, os enfermeiros têm sido vítimas da violência física ${ }^{(15)}$.

A violência contra enfermeiros e demais profissionais da saúde tornou-se desafio para o sistema de saúde brasileiro e trouxe sérias consequências a estes profissionais, provocando angústia, sofrimento mental, palpitações, desordem no sono, estresse, depressão, danos na audição, dores de cabeça, ansiedade, medo dos pacientes e de seus familiares e, até mesmo culminando em suicídio ${ }^{(16)}$. Diante dessa realidade e considerando os índices de enfermeiros agredidos em seus postos de trabalho, os gestores e autoridades devem repensar e investir em políticas de conscientização contra a violência no trabalho dessa categoria.

Outro aspecto das condições de trabalho relacionado aos riscos psicossociais e muito mencionado nesta investigação, diz respeito aos equipamentos de trabalho e recursos materiais insuficientes e/ou com manutenção falha, que também foi percebido em outros estudos como fator desencadeante de estresse nos profissionais ${ }^{(9-10)}$. Na maioria das unidades, os enfermeiros compram e arcam com os custos do reparo dos materiais e ainda não possuem veículo para transporte nas visitas domiciliares, mesmo quando o cliente mora distante da área da unidade, o que leva a maioria dos profissionais a utilizarem seus próprios veículos, sem repasse de verba.

A falta de recursos materiais e estrutura física inadequada são realidades na ESF. Pesquisadores já evidenciaram problemas como ausência de recursos para atendimento de primeiros socorros, e dificuldades de acesso à internet $\mathrm{e}$ ao telefone para desenvolver ações de atendimento individual com privacidade ${ }^{(17)}$.

A literatura aponta ainda que a manutenção dos Programas de Saúde da Família gera um custo elevado para o governo, onde os recursos repassados aos municípios pela União e Estados são insuficientes para cobrir as demandas dos serviços de saúde na $\mathrm{ESF}^{(18)}$. Entretanto, para os enfermeiros desempenharem suas funções com êxito e eficácia, precisam de uma estrutura física e recursos suficientes, a fim de atender todas as demandas do serviço.

É importante que os gestores tenham consciência e repensem a implementação de políticas de garantia de tais recursos e sua manutenção, haja vista o impacto dessa temática na saúde pública brasileira. Se os gestores conhecerem e compreenderem os propósitos e a realidade da ESF em cada localidade, haverá uma transformação na destinação dos recursos, com consequente melhora das condições de trabalho de toda a equipe, uma vez que a organização da Atenção Primária à Saúde se desenvolve a partir do contexto político, social e cultural de cada país ${ }^{(19)}$.

Além do ambiente e equipamentos adequados, são necessários profissionais em número e qualidade para executarem suas funções de forma integral. O número insuficiente de profissionais na ESF e o desvio de função laboral revelam outro importante risco psicossocial. Nesta linha de pensamento, outros estudos identificaram a ESF com quadro profissional incompleto e ausência significativa de Agentes Comunitários de Saúde (ACS) e médicos. A ausência destes profissionais pode ser justificada pela necessidade de abrir novos processos de contratação, insatisfação com a remuneração salarial e afastamento do local de trabalho por motivo de licença saúde ${ }^{(20)}$.

A falta do ACS se configura como grande problema em todas as ESF do município pesquisado. As poucas equipes que contemplam todos os ACS preconizados pelo Ministério da Saúde ainda encontram impasses, pois sempre houve desvio de função desse profissional para a recepção. Os resultados obtidos também demonstraram equipes funcionando somente com dois ACS, um médico e um enfermeiro. Esse fato pode ser corroborado à pesquisa que revelou excesso de funções previstas para o ACS na ESF, além da falta de clareza de suas atribuições ${ }^{(21)}$. Esses dados nos levam a refletir sobre a forma como essa estratégia foi implantada e como foram selecionados os profissionais, pois são sabidamente conhecidos a importância e o nível de responsabilidade desse setor na reorganização da Atenção Básica no Brasil.

Os recursos humanos na Saúde da Família, especialmente com relação à equipe de Enfermagem e sobrecarga de trabalho, constituem um desafio e risco, haja vista a necessidade da população usuária das redes de atenção. A literatura 
aponta que o ritmo do trabalho decorrente das diversas atribuições do enfermeiro na ESF e a alta demanda desse setor podem gerar facilmente sobrecarga de trabalho, que é um risco psicossocial evidenciado por estudiosos da temática ${ }^{(22)}$. Para o perfeito funcionamento da ESF, é necessário reestruturar e formar recursos humanos suficientes para atender as demandas da saúde pública ${ }^{(23)}$.

Vale destacar que no cotidiano dos participantes, ultrapassar a carga horária diária de trabalho é comum, acarretando o sentimento de esgotamento físico, mental, desvalorização e esquecimento pelo sistema. Acerca desta afirmação, pesquisadores apontam a relação entre cargas horárias de trabalho extensas na enfermagem, associada às más condições laborais, pouca autonomia, muita responsabilidade e baixa valorização, que geram elevada demanda física e emocional $^{(24-25)}$. A sobrecarga de atividades pode acarretar outros problemas para os enfermeiros, por exemplo, o desequilíbrio ao conciliar o trabalho e a família.

A falta de equilíbrio entre as exigências do lar e do trabalho, e o pouco tempo dispensado para a família também foram percebidos pelos enfermeiros como um risco psicossocial. Os principais fatores que interferem na relação trabalho-família são o acúmulo de horas extras e tarefas e a transferência de trabalho para casa.

Em consonância com essa pesquisa, o risco psicossocial interface trabalho-família foi encontrado em outras evidências com o profissional enfermeiro. Esse risco representa importante indicador capaz de adoecer os trabalhadores ${ }^{(26)}$. Nesta vertente, a literatura aponta a importância do equilíbrio entre a interface trabalho/família e da qualidade do ambiente laboral enquanto mediador para o estabelecimento de relações familiares positivas e o desenvolvimento socioemocional e escolar dos filhos ${ }^{(27)}$. Além disso, é importante criar políticas públicas para eliminar esse conflito, com fins de garantir condições de trabalho dignas e equilibradas aos enfermeiros, pois esse profissional encontra-se muito exposto a esse e aos demais riscos psicossociais.

Em continuidade, reforça-se a necessidade de profissionais em quantidade e qualidade para as equipes das ESF. É imprescindível que a União, Estados e Munícipios garantam o número mínimo de profissionais para um contexto de trabalho digno e seguro. Gestores devem repensar a organização do trabalho e oferecer condições laborais adequadas e recursos humanos em quantidade e qualidade para o cuidado efetivo na Atenção Básica.

Um dos caminhos para diminuir os riscos psicossociais é elaborar e implementar estratégias individuais e organizacionais. Os discursos dos enfermeiros revelaram ausência de atividades ou ações estratégicas oferecidas pela coordenação das equipes da ESF, ao mesmo tempo em que foram relatadas estratégias individuais, como a prática de atividade física e meditação, auxiliando em situações estressantes do cotidiano.
As organizações têm a corresponsabilidade de manter a saúde do seu trabalhador. Portanto, devem elaborar estratégias para colaborar com estes profissionais no alívio dos riscos a que estão expostos no cotidiano do seu trabalho e consequentemente, contribuir para um cuidado de qualidade. Quando estes profissionais possuem apoio e condições satisfatórias no trabalho, seu desempenho melhora, pois se sentem motivados e esse é um fator determinante para uma assistência integral de qualidade ao usuário.

Em consonância com o apresentado, a literatura descreve estratégias individuais e institucionais para gerenciar os riscos psicossociais em outros níveis de atenção à saúde, como exercício físico, cinema, apoio espiritual, busca por terapias, diálogo com familiares, música, leitura, viagens, passeios, programas de educação continuada, sistema eficaz de comunicação interna, planos de cargos e salários, entre outros ${ }^{(15)}$.

Essa pesquisa apresentou a limitação de ter sido realizada com um número pequeno de profissionais em determinada região do país. Daí a importância de realizar outros estudos para ampliar a quantidade e abrangência para outros Estados, com intuito de verificar se há divergência na percepção dos riscos e repensar estratégias para gerenciamento. A pesquisa descreveu diversos riscos psicossociais essenciais para estimular a reflexão de gestores, centros formadores em saúde e enfermeiros no sentido de criar estratégias para enfrentá-los no cotidiano laboral.

\section{CONCLUSÃO}

A prática do enfermeiro no contexto da saúde da família reúne complexas situações de trabalho e, dependendo da forma como esta prática é organizada, o profissional acaba exposto a riscos psicossociais que podem acarretar o seu sofrimento psíquico. A identificação destes riscos reforça a presença de problemas nas políticas públicas de gestão de pessoas e recursos na área da saúde. Deve haver atenção para a criação de ambientes adequados e preparo dos profissionais para responder às necessidades de saúde da população.

Existe um cenário preocupante na ESF, onde o enfermeiro não possui suporte institucional para enfrentar possíveis problemas nas condições de trabalho, o que interfere negativamente. Aliado a isso, os trabalhadores recorrem a estratégias individualmente para aliviar a presença dos riscos ocupacionais presentes. A União, Estados e Municípios, devem repensar a sua responsabilidade em garantir o pleno funcionamento da ESF, incluindo a manutenção de condições de trabalho adequadas para os profissionais que compõem esse nível de Atenção. Os centros formadores devem repensar suas estruturas curriculares, incluindo metodologias ativas no ensino-aprendizagem de futuros profissionais, preparando um trabalhador com perfil de competências específicas para responder às demandas do mercado de trabalho, em especial da ESF.

RESUMO

Objetivo: Analisar os riscos psicossociais relacionados ao trabalho do enfermeiro da Saúde da Família e as estratégias de gerenciamento para minimizá-los. Método: Estudo exploratório, qualitativo, com enfermeiros das equipes da Estratégia da Saúde da Família. A coleta de dados ocorreu em julho de 2018 por entrevistas semiestruturadas e posteriormente foi realizada análise temática indutiva. Resultados: Participaram 18 enfermeiros. Foram identificados riscos psicossociais relacionados ao contexto de trabalho: capacitação 
profissional insuficiente, relacionamento interpessoal comprometido, interface trabalho-família e violência psicológica; e relacionados ao conteúdo de trabalho: equipamento de trabalho insuficiente, déficit de recursos humanos e carga de trabalho extensa. Além disso, foram identificadas estratégias de gerenciamento destes riscos, tais como recorrer à família, espiritualidade, música e leitura. Conclusão: O estudo deve provocar a reflexão dos enfermeiros acerca dos riscos que podem estar presentes no seu trabalho, além de contribuir para preencher lacunas na produção de conhecimento da área abordada. Pode ainda, fornecer subsídios para que os gestores de saúde conheçam os riscos psicossociais aos quais estão expostos estes profissionais, na perspectiva de adotar providências.

\section{DESCRITORES}

Enfermagem Familiar; Estratégia Saúde da Família; Riscos Ocupacionais; Enfermagem de Atenção Primária; Saúde do Trabalhador.

\section{RESUMEN}

Objetivo: Analizar los riesgos psicosociales relacionados con el trabajo de los enfermeros de la Salud de la Familia y las estrategias de gestión para minimizarlos. Método: Estudio exploratorio, cualitativo con enfermeros de los equipos de la Estrategia de Salud de la Familia. La recolección de datos fue en julio de 2018 a través de entrevistas semiestructuradas y, posteriormente, se realizó un análisis temático inductivo. Resultados: Participaron 18 enfermeros. Se identificaron riesgos psicosociales relacionados con el contexto laboral: capacitación profesional insuficiente, relaciones interpersonales comprometidas, interfaz trabajo-familia y violencia psicológica; y relacionados con el contenido del trabajo: equipo de trabajo insuficiente, falta de recursos humanos y gran carga de trabajo. Además, se identificaron estrategias para manejar estos riesgos, como el uso de la familia, la espiritualidad, la música y la lectura. Conclusión: El estudio debería provocar la reflexión de los enfermeros sobre la eventual presencia de riesgos en su trabajo y contribuir a llenar las brechas de producción de conocimiento en el área. También proporciona subsidios para que los administradores de salud conozcan los riesgos psicosociales a los que están expuestos estos profesionales, con miras a adoptar medidas.

\section{DESCRIPTORES}

Enfermería de la Familia; Estrategia de Salud Familiar; Riesgos Laborales; Enfermería de Atención Primaria; Salud Laboral.

\section{REFERÊNCIAS}

1. Motta LCS, Siqueira-Batista R. Estratégia Saúde da Família: clínica e crítica. Rev Bras Educ Med. 2015;39(2):196-207. DOI: http://dx.doi. org/10.1590/1981-52712015v39n2e00912014

2. Brasil. Ministério da Saúde. Portaria n. 2.436, de 21 de setembro de 2017. Aprova a Política Nacional da Atenção Básica estabelecendo a revisão de diretrizes para a organização da Atenção Básica, no âmbito do Sistema Único de Saúde. Brasília; 2017.

3. Pires DEP, Machado RR, Soratto J, Scherer MA, Gonçalves ASR, Trindade LL. Nursing workloads in family health: implications for universal access. Rev Latino Am Enfermagem. 2016;24:e2677. DOI: http://dx.doi.org/10.1590/1518-8345.0992.2682

4. Agência Europeia para a Segurança e a Saúde no Trabalho. Guia eletrônico sobre a gestão do stresse e dos riscos psicossociais no local de trabalho [Internet]. Luxemburgo; 2018 [citado 2018 dez. 12]. Disponível em: https://osha.europa.eu/pt/tools-and-publications/e-guidemanaging-stress-and-psychosocial-risks

5. Fernandes C, Pereira A. Exposição a fatores de risco psicossocial em contexto de trabalho: revisão sistemática. Rev Saúde Pública. 2016;50:24. DOI: 10.1590/S1518-8787.2016050006129

6. Fernandes DM, Marcolan JF. Work and depression symptoms in Family Health Strategy nurses. SMAD Rev Eletr Saúde Mental Álcool Drog. 2017;13(1):37-44. https://dx.doi.org/10.11606/isse.1806-6976.v13i1p37-44

7. Horta NC, Moreira DA, Aguiar KT, Morais JL. Potenciais de desgaste biopsíquico nos profissionais de enfermagem que atuam na Estratégia de Saúde da Família: percurso acadêmico. 2014;4(7):105-19. DOI: https://doi.org/10.5752/P.2236-0603.2014v4n7p105-119

8. Camelo SHH, Chaves LDP, Silva VLS, Angerami ELS. Riscos psicossociais em equipes de saúde da família: carga, ritmo e esquema de trabalho. Rev Enferm UERJ [Internet]. 2012 [citado 2018 dez. 12];20(6):733-8. Disponível em: https://www.e-publicacoes.uerj.br/index. php/enfermagemuerj/article/view/5990

9. Araújo ST, Penaforte KL. Riscos psicossociais relacionados ao trabalho: percepção dos profissionais de enfermagem. Rev Enferm UFPE on line. 2016;10(11):3831-9. DOI: 10.5205/reuol.9881-87554-1-EDSM1011201603

10. Chagas D. Riscos psicossociais no trabalho: causas e consequências. Rev Psicol. 2015;2(1):439-46. DOI: http://dx.doi.org/10.17060/ ijodaep.2015.n1.v2.24

11. Cherques HRT. Saturação em pesquisa qualitativa: estimativa empírica de dimensionamento. Revista PMKT. 2009;3:20-27.

12. Braun V, Clarke V. Using thematic analysis in psychology. Qual Res Psychol. 2006;3(2):77-101. DOI: 10.1191/1478088706qp063oa

13. Cunha $P$, Meneses R, Oliveira MC. Gestão de conflitos na área da saúde: uma proposta de reflexão. Arq Med [Internet]. 2013 [citado 2018 dez. 12];27(3):132-4. Disponível em: http://www.scielo.mec.pt/scielo.php?script=sci_arttext\&pid=S0871-34132013000300006

14. Silva IS, Arantes CIS. Power relations in the family health team: focus on nursing. Rev Bras Enferm. 2017;70(3):607-15. DOI: http://dx.doi. org/10.1590/0034-7167-2015-0171

15. Scozzafave MCS, Camelo SHH, Soares MI, Rocha FLR, Gaioli CCLO, Leal LA. Violence as psychosocial risk in the work of psychiatric nurses and management strategies. Int Arch Med. 2017;10(43):1-10. DOI: https://doi.org/10.3823/2313

16. Lima GHA, Sousa SMA. Psychological violence in the nursing work. Rev Bras Enferm [Internet]. 2015;68(5):535-41. DOI: http://dx.doi. org/10.1590/0034-7167.2015680508i

17. Moreira KS, Lima CA, Vieira MA, Costa SM. Assessment of infrastructure of family health units and equipment used in primary care actions. Cogitare Enferm. 2017;22(2):e51283. DOI: http://dx.doi.org/105380/ce.v22i1.51283

18. Costa NR. The family health strategy: primary health care and the challenge of Brazilian metropolises. Ciênc Saúde Coletiva. 2016;21(5):1389-98. DOI: http://dx.doi.or/10.1590/1413-81232015215.24842015 
19. Arantes LJ, Shimizu HE, Merchán-Hamann E. Organizational processes in the Family Health Strategy: an analysis conducted by nurses. Acta Paul Enferm. 2016;29(3):274-81. DOI: http://dx.doi.org/10.1590/1982-0194201600039

20. Eslabao AD, Coimbra VCC, Kantorski LP, Pinho LB, Santos EO. Mental health care network: the views of coordinators of the Family Health Strategy (FHS). Rev Gaúcha Enferm. 2017;38(1):e60973. DOI:http://dx.doi.org/10.1590/1983-1447.2017.01.60973

21. Alonso CMDC, Beguin PD, Duarte FJCM. Work of community health agents in the Family Health Strategy: meta-synthesis. Rev Saúde Pública. 2018;52(14):1-13. DOI: https://doi.org/10.5935/1676-4285.200535

22. Cox T, Griffiths A, Rial-González E. Research on work-related stress [Internet]. Luxembourg: European Agency for Safety and Health at Work; 2000 [cited 2018 Dec 12]. Available from: https://osha.europa.eu/en/publications/report-research-work-related-stress

23. Gonçalves CR, Cruz MT, Oliveira MP, Morais AJD, Moreira KS, Rodrigues CAQ, et al. Recursos humanos: fator crítico para as redes de atenção á saúde. Saúde Debate [Internet]. 2014 [citado 2018 dez. 12];38(100):26-34. DOI: 105935/0103-104.20140012

24. Silva Neto JA, Torres CRD, Feitosa KVA, Gouveia MTO, Torres JRD. Aspectos jurídicos da jornada de trabalho em enfermagem: reflexão teórica. Rev Enferm UFPI [Internet]. 2015 [citado 2018 dez. 12];4(3):95-8. Disponível em: http://www.ojs.ufpi.br/index.php/reufpi/article/ view/2368/pdf

25. Lala AL, Sturzu LM, Picard JP, Druot F, Grama F, Bobirnac G. Coping behavior and risk and resilience stress factors in French regional emergency medicine unit workers: a cross-sectional survey. J Med Life. 2016;9(4):363-8. DOI: 10:22336/jml.2016.0408

26. Scozzafave MCS, Leal LA, Soares MI, Henriques SH. Psychosocial risks related to the nurse in the psychiatric hospital and management strategies. Rev Bras Enferm [Internet]. 2019;72(4):834-40. DOI: http://dx.doi.org/10.1590/0034-7167-2017-0311

27. D’Affonseca SM, Cia F, Barham EJ. Trabalhadora feliz, mãe feliz? Condições de trabalho que influenciam na vida familiar. Psicol Argum. 2014;32(76):129-38. DOI: 10.7213/psicol.argum.32.076.AO08 